\title{
Sister chromatid cohesion in silent chromatin: each sister to her own ring
}

\author{
Julie Huang ${ }^{2}$ and Danesh Moazed ${ }^{1}$ \\ Department of Cell Biology, Harvard Medical School, Boston Massachusetts 02115, USA
}

The proper transmission of genetic material is crucial for the growth and survival of all organisms. Each time a eukaryotic cell divides, it must faithfully produce two copies of each chromosome, known as sister chromatids, and accurately distribute them between two daughter cells. To ensure their faithful transmission, newly replicated sister chromatids must be held together long enough to allow proper bipolar attachment to microtubules and alignment on the metaphase spindle. Cohesion of sister chromatids is mediated by cohesin, an evolutionarily conserved, multiprotein complex that is associated with chromosome arms and is highly enriched at the centromeres of chromosomes (Hagstrom and Meyer 2003; Losada and Hirano 2005; Nasmyth and Haering 2005). How the cohesin complex is recruited to chromosomes and how it holds sister chromatids together are of fundamental importance to cell biology and are areas of intense investigation.

Centromeres of most eukaryotes are composed of highly repetitive DNA sequences and are packaged into heterochromatin, a physically condensed chromatin structure, which is transcriptionally silent and refractory to recombination (Karpen and Allshire 1997; Bernard and Allshire 2002; Henikoff and Dalal 2005). Ample genetic evidence from fission yeast, Drosophila, and mammalian cells indicates that heterochromatin formation is important for proper centromere function, as mutations that perturb heterochromatin at these regions also cause defects in cohesion of sister centromeres and chromosome missegregation (Allshire et al. 1995; Kellum and Alberts 1995; Ekwall et al. 1996, 1999; Peters et al. 2001). At the same time, it is clear that heterochromatin is found at a variety of other loci in eukaryotic genomes such as the silent-mating type loci in both budding and fission yeasts and throughout the inactive $\mathrm{X}$ chromosome in mammals. Is there something unique about centromeric heterochromatin or do other heterochromatic regions also mediate chromosome cohesion? What additional roles might cohesin have in chromosome organization at these sites? In a recent issue of Genes \& De-

Correspondence.

${ }^{1}$ E-MAIL danesh@hms.harvard.edu; FAX (617) 432-1144.

${ }^{2}$ E-MAIL Julie_Huang@hms.harvard.edu; FAX (617) 432-1144.

Article and publication are at http://www.genesdev.org/cgi/doi/10.1101/ gad.1398106. velopment, Gartenberg and colleagues (Chang et al. 2005) investigate the relationship between silent chromatin and chromosome cohesion in budding yeast. Using a series of elegant experiments, Chang et al. (2005) discover that cohesin is in fact recruited to silent chromatin in this yeast. Their results not only provide unexpected insight into the mechanism of cohesin association with silent chromatin, but also impact current models of how the cohesin complex mediates sister chromatid cohesion.

Cohesin is a stable complex that consists of four subunits, Smc1, Smc3, Scc1/Mcd1 (also known as kleisin), and Scc3, all of which are conserved from yeast to humans (Fig. 1; for review, see Losada and Hirano 2005). Interactions between the subunits, as revealed by immunoprecipitation assays, are consistent with a complex that forms a large ring structure (Gruber et al. 2003), and purified cohesin complexes visualized by electron microscopy can form ring-like structures (Haering et al. 2002). During G1 of the cell cycle, the complex is loaded onto unreplicated DNA. As S-phase progresses, cohesin, with the help of accessory proteins, establishes cohesion between newly replicated sister chromatids, which is maintained through G2 and part of M phase. Sister chromatids are segregated during the metaphase-to-anaphase transition as a result of the cleavage of the $\mathrm{Scc} 1 / \mathrm{Mcd} 1$ subunit by the cysteine protease separase, thereby destabilizing the cohesin complex (Gerton 2005; Losada and Hirano 2005). Based on these and other observations, it has been proposed that the cohesin complex forms a giant ring that embraces replicated DNA, releasing sister chromatids once Sccl/Mcdl is cleaved to open the ring (Fig. 1; Haering et al. 2002). According to this model, cohesin does not bind chromatids physically, but rather, traps paired sisters topologically within the ring (Haering et al. 2002; Gruber et al. 2003; Nasmyth 2005). In an alternative model, rings of cohesin, rather than encircling DNA, interact with each other and with one sister chromatid apiece to snap sisters together (Milutinovich and Koshland 2003; Huang et al. 2005).

Genome-wide mapping studies in Saccharomyces cerevisiae indicate that cohesin associates with discrete sites along chromosome arms, but is most enriched at centromeres (Blat and Kleckner 1999; Tanaka et al. 1999; Laloraya et al. 2000; Lengronne et al. 2004; Weber et al. 2004). In both fission yeast and metazoans, cohesin as- 


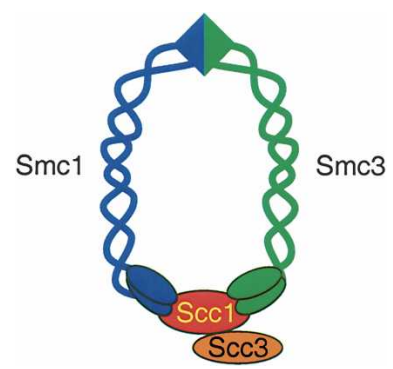

Figure 1. Subunit composition of the cohesin complex. The Smc1, Smc3, Scc1/Mcd1, and Scc3 subunits are shown in blue, green, red, and orange, respectively.

sociation is lost first from the arms during prophase and then later from centromeres when sister chromatids finally separate. In Schizosaccharomyces pombe, centromeric cohesion may be distinguished from arm cohesion in part by the presence of the heterochromatin structural protein, Swi6, which binds cohesin and is required for its recruitment to centromeres (Bernard et al. 2001; Nonaka et al. 2002). Since Swi6 has homologs (HP1 family proteins) in both Drosophila and humans, a similar mechanism may also apply to metazoans. In addition, cohesin complexes with altered subunit compositions that carry out differing functions at the pericentric and arm regions of meiotic chromosomes have also been identified (Kitajima et al. 2003). These studies raise the possibility that alternative mechanisms and accessory factors may exist for the recruitment and perhaps the attachment of cohesin to different chromosome regions.

However, in budding yeast, centromeres do not evince characteristics of heterochromatin (Sharp et al. 2003). In this organism, heterochromatin is found at chromosome arm regions such as the silent mating-type cassettes, telomeres, and at the ribosomal RNA genes (rDNA). Silencing at the mating-type cassettes regulates haploid cell identity by ensuring that only one of two alleles at the mating-type locus $(M A T)$ is expressed. On opposite ends of the same chromosome as the expressed MAT locus are the silent mating-type cassettes, $H M L$ and $H M R$, respectively. Each locus consists of a fully functional but silent copy of one of the two mating-type alleles, allowing cells to switch mating-type through a mechanism similar to gene conversion. To ensure that the silent loci are maintained in a repressed state until needed, $H M L$ and $H M R$ are each flanked by cis-regulatory elements, the E and I silencers. These sequences are bound by various combinations of DNA-binding initiation factors, which recruit the dedicated silencing factors, Sir2, Sir3, and Sir4. Sir3 and Sir4 are histone-binding proteins, while Sir2 is a highly conserved protein deacetylase, whose activity is required for silencing and likely targets acetylated histones that package DNA in chromatin. Deacetylation ultimately results in the cooperative association of the Sir proteins with initiation factors, histones, and each other, leading to their spreading along DNA to generate the refractory chromatin structure of heterochromatin (Rusche et al. 2003; Moazed et al. 2004). Prior to the work described by Chang et al. (2005), none of the silent regions of budding yeast had been directly tested for a role in sister chromatid cohesion.

Sister chromatid cohesion is typically assayed visually, such as by analyzing concentrated, fluorescent "dots" emanating from sister chromatids, each of which is bound by a bacterial DNA-binding protein fused to green fluorescent protein (GFP) (Straight et al. 1996; Michaelis et al. 1997). While such methods have been invaluable for studying overall chromosomal cohesion, they are not especially conducive to studying the cohesion status of a particular locus. Since adjacent sites can gain or lose cohesin binding independently of each other (Megee et al. 1999; Glynn et al. 2004), defects in cohesion at a single site can be compensated for or masked by cohesin binding at neighboring regions, thus remaining undetected.

To circumvent this problem, Gartenberg and colleagues adapted an approach used successfully to address several other key questions about the nature of heterochromatin, such as whether maintenance of silent domains continuously requires silencers, whether the establishment of silencing requires DNA replication, and how silencing influences nuclear architecture (Holmes and Broach 1996; Bi and Broach 1997; Cheng and Gartenberg 2000; Kirchmaier and Rine 2001; Li et al. 2001; Gartenberg et al. 2004). Here, Chang et al. (2005) generated yeast strains in which site-specific recombination could be used to excise the HMR locus away from the chromosome. The excised circles included silencer elements that, as previously observed, remain functional after excision (Bi and Broach 1997; Cheng and Gartenberg 2000; Kirchmaier and Rine 2001). The circles also contained an array of bacterial $\mathrm{LacO}$ sequences adjacent to the silent locus, allowing cohesion of excised circles to be monitored through the visualization of ectopically expressed LacI-GFP protein bound to the arrays (Fig. 2). Cells were allowed to duplicate their genomes, arrested

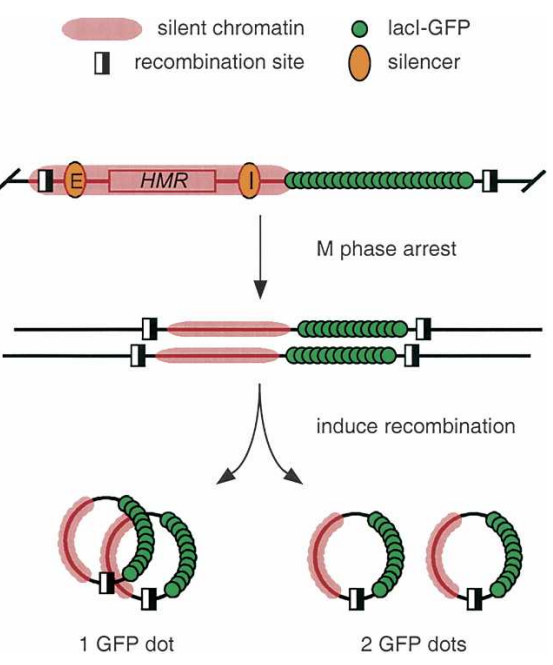

Figure 2. Experimental design of the excisable $H M R$ locus. See text for details. Adapted from Chang et al. (2005). 
in $M$ phase, and induced to express recombinase, thus producing newly replicated and excised "sister chromatin circles". Similar to what has been observed for linear chromatids, these circles emit a single fluorescent dot when associated, but two dots when disassociated. This experimental design allows the study of cohesion at a site independent of its chromosomal context, and a variety of hypotheses can be tested simply by altering the cis-element sequences on the rings or manipulating the conditions under which excision occurs.

Using this strategy, Chang et al. (2005) first discovered that upon excision, replicated $H M R$ circles remained cohered. One important question to address was whether this pairing was due to the heterochromatic state of the circles. To test this, key silencing proteins such Sir3 or Sir4 were removed prior to ring excision. This removal results in a dramatic reduction in colocalization of excised circles. Similarly, inhibition of Sir2 deacetylase activity by splitomicin, a small molecule inhibitor, prior to excision also reduces ring pairing. In contrast, a centromeric plasmid that does not contain the $H M R$ cassette remains cohered in the absence of Sir3, indicating that silencing proteins play a role in cohesion specific to the silent mating-type locus. Corruption of silencer elements necessary for silent chromatin assembly also leads to a loss of pairing upon excision, supporting the idea that the effect of silencing on cohesion occurs in cis and is not due to a loss of transcriptional silencing elsewhere in the genome. Significantly, in every case that transcriptional silencing was abrogated but the $H M R$ locus was not excised from its host chromosome, loss of sister chromatid cohesion was rarely observed. Again, this requirement for silencers indicates that loss of cohesion at silent domains does not affect global sister chromatid cohesion and further underscores the importance of experimental design when investigating the pairing activity of a specific region of the genome. Although silent chromatin is involved in cohesion at centromeric heterochromatin in fission yeast, Drosophila, and mammalian cells (Karpen and Allshire 1997; Bernard and Allshire 2002), this is the first demonstration in budding yeast that disruption of silencing results in loss of cohesion at a specific site.

Gartenberg and colleagues (Chang et al. 2005) next explored what other factors are required for cohesion of $H M R$ circles. A logical candidate was the cohesin complex, often characterized as the "glue" that holds sister chromatids together. Furthermore, genome-wide mapping of cohesin-binding sites in budding yeast by chromatin immunoprecipitation (ChIP) had indicated that cohesin is enriched at sites both within and flanking the silent mating-type loci (Laloraya et al. 2000; Glynn et al. 2004). Chang et al. (2005) find that in cells expressing temperature-sensitive mutations of Scc1/Mcd1 or Smc3, two subunits of cohesin, pairing of both intact chromosomes and silent chromatin circles is dramatically reduced. For the chromatin circles, deletion of the silencing factor Sir3 in these cohesin mutant cells does not increase the percentage of cells displaying separated fluorescent dots, indicating that cohesin and silencing pro- teins act in the same pathway to establish cohesion. Finally, consistent with these findings, ChIP experiments indicate that the association of the Scc1/Mcd1 subunit of the cohesin complex with the unexcised $H M R$ locus requires functional silencing. These results therefore suggest that silent chromatin is indeed required for the recruitment of cohesin to the $H M R$ locus and the maintenance of cohesin-dependent cohesion of excised $H M R$ circles.

Gartenberg and colleagues (Chang et al. 2005) next examined the fate of cohesins after their recruitment to the $H M R$ locus using two approaches. In one set of experiments, the recombination sites flanking the $H M R$ locus were repositioned, resulting in excision of $H M R$ away from its $\mathrm{E}$ and I silencer elements, which are required continuously for the maintenance of silencing on chromatin circles (Holmes and Broach 1996; Cheng and Gartenberg 2000). Under these conditions, the chromatin on the circles initially is silent but becomes derepressed after excision. In a second set of experiments, the silencer elements were included on the looped-out cassette, but after excision, silencing was abolished by the addition of the Sir2 deacetylase inhibitor, splitomicin. The presence of Sccl/Mcdl at $H M R$ sequences was determined by ChIP under both nonexcising and excising conditions. When the $H M R$ locus remained on a linear chromosome, inactivation of silencing resulted in a concomitant loss of Scc1/Mcd1 association, arguing that silent chromatin is required for the initial targeting as well as the maintenance of cohesin at $H M R$.

Strikingly, Chang et al. (2005) discovered that even when silencing is disrupted by either the removal of silencers or the addition of splitomicin, the levels of Scc1 associated with excised $H M R$ circles remains unchanged. How does excision prevent the dissociation of cohesin from HMR circles? The observations of Chang et al. strongly support the idea that the cohesin complex does form a ring that encircles DNA, as proposed by Nasmyth and colleagues (see below) (Haering et al. 2002). After the cohesin complex is recruited to silent chromatin, it essentially becomes trapped there once the chromosome region is circularized. Apparently, lateral diffusion around the excised circle does not diminish the observed binding of cohesin to HMR in ChIP assays. Therefore, although silent chromatin is required for both the initiation and maintenance of cohesin at the chromosomal HMR locus, cohesin is artificially trapped by circularization of the $H M R$ cassette, irrespective of silencing.

These results are in agreement with recently published findings from an elegant set of experiments designed to test whether the cohesin complex interacts with DNA as a topological ring (Ivanov and Nasmyth 2005). In this study, circular plasmids containing a centromere and an origin of DNA replication were transformed into budding yeast and permitted to undergo duplication and subsequent loading of cohesins. The plasmids also contained an array of bacterial Tet operator sequences bound by affinity-tagged Tet repressors, allowing rapid purification from whole-cell extracts. Puri- 
fication of these plasmids results in coprecipitation of cohesin complexes and, conversely, immunoprecipitation of cohesin subunits results in coprecipitation of plasmid DNA. Importantly, precipitated complexes in which the plasmid DNA is linearized by restriction enzymes no longer contain cohesin. Similarly, complexes in which the cohesin ring is opened by cleavage of one of its subunits are not associated with the plasmid DNA. These and other experiments convincingly demonstrate that cohesin does indeed behave as a ring that entraps DNA. Since the circular DNA in the biochemical experiments does not contain silent chromatin, the combined results of these two studies argue that cohesin complexes can associate with both active and inactive regions of the yeast genome in the form of topological rings.

An attractive aspect of the ring model of cohesion proposed by Nasmyth and colleagues (Haering et al. 2002; Ivanov and Nasmyth 2005) is that it provides a straightforward mechanism for holding sister chromatids together following replication. By embracing paired chromatids topologically, cohesin is not required to stably associate with specific DNA sequences or proteins. In support of this model, several studies indicate that cohesins can exhibit significant lateral mobility along DNA (Glynn et al. 2004; Lengronne et al. 2004; Ivanov and Nasmyth 2005). Furthermore, the diameter of the cohesin ring appears to be $\sim 40 \mathrm{~nm}$, wide enough to hold two 10-nm nucleosomal chromatin fibers (Haering et al. 2002). A key predication of this model is that loading of cohesin onto replicated chromosome rings should be sufficient to maintain pairing between them. Chang et al. (2005) were also able to test this aspect of the ring model at silent chromatin by visually monitoring the behavior of LacI-GFP bound to HMR circles to determine whether trapped cohesins were competent for cohesion.

Surprisingly, maintenance of functional pairing of $H M R$ circles was found to be dependent on the continuous presence of silent chromatin, as had been observed for the linear HMR locus. After excision, disruption of silencing by removal of silencers or the addition of splitomicin perturbs cohesion of the paired $H M R$ circles, despite the continued association of cohesin. Therefore, although silencing is not necessary for physically trapping cohesin on circular DNA, it is indeed required for its cohesion activity. One important implication from this finding is that accessory factors may be required to mediate cohesin function at heterochromatin and perhaps other chromosomal regions. Although a physical interaction between the Sir proteins and cohesin has not been reported to date, such an interaction may be chromatin-dependent and difficult to detect. It remains possible that the Sir proteins directly bind to and recruit cohesins, as is the case with the heterochromatin protein Swi6, which associates with and recruits cohesin to centromeres in S. pombe (Nonaka et al. 2002).

The work by Chang et al. (2005) suggests a new model for cohesin function, in which cohesins encircle only one, rather than both sister chromatids (Fig. 3). This model of cohesin association shares the circular topology
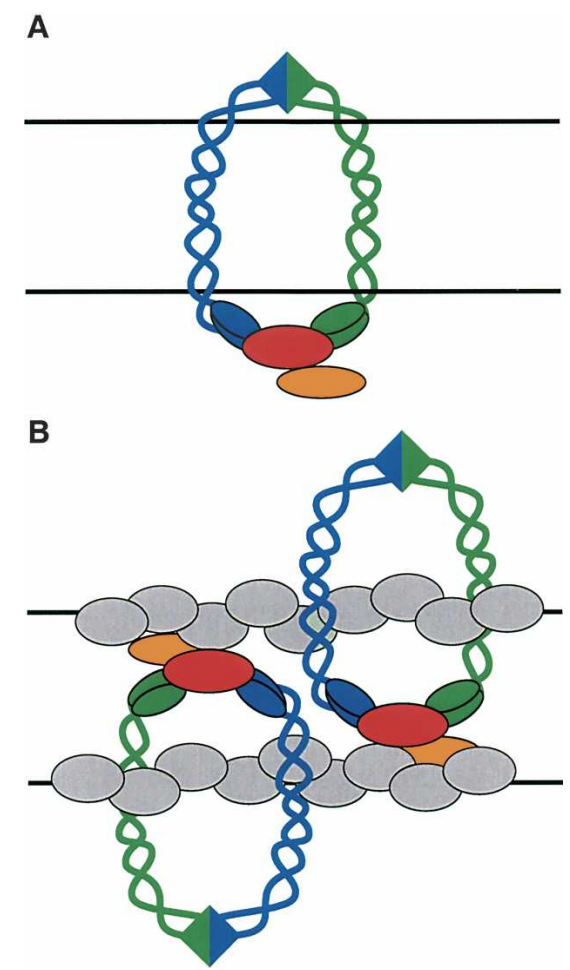

Figure 3. Models for sister chromatid cohesion. Gray ovals represent silencing proteins or accessory factors. See the legend for Figure 1 for subunit labels and the text for details.

and lateral mobility suggested by the ring model, but necessitates an altered mechanism of sister chromatid pairing. Chang et al. suggest that cohesin rings encircling one chromatid may interact with cohesin rings embracing its sister chromatid. It is possible that this mechanism of cohesin binding is specific to silent regions, evolving in response to a chromatin fiber whose diameter is enlarged due to higher order chromatin compaction and the presence of heterochromatin proteins. Whether this "single chromatid" model of cohesin function applies to other chromosomal sites is not yet known. Thus far, immunoprecipitation experiments have failed to uncover interactions between individual cohesin rings, arguing against widespread cohesion mediated by a single chromatid ring model, which requires direct or indirect physical interactions between individual cohesin rings to generate pairing. But the ultimate answer to this important question is likely to come from the application of the DNA excision strategy used by Chang et al. to sites of cohesin association with other chromosome regions.

Finally, Chang et al. (2005) observed that while silencing is required for cohesion, the reverse is not true, at least with respect to maintenance of silencing. This observation, combined with their finding that loss of silencing has no obvious detrimental affect on cohesion at nonheterochromatic sites, suggests an increased need for cohesion at silent loci, but what this function is remains unknown. One possibility is that cohesin is needed to maintain the genomic stability at sites of heterochroma- 
tin, perhaps due to an elevated risk of genomic rearrangement, as has been observed in $S$. pombe (Nonaka et al. 2002). In this organism, a relatively similar silent mating-type region has been shown to recruit cohesin, but once again, cohesin is not required for silencing. However, defects in cohesin increase the frequency at which silent alleles of the mating type, called mat2 and mat3, improperly integrate into the active mat1 locus, interfering with mating-type switching. These rearrangements may occur because sister chromatids are not held in proper alignment by cohesion, leading to mitotic recombination between regions that share significant sequence homology, as is the case with mat1 and the silent mat $2 / 3$ loci. It would be interesting to determine whether cohesin plays a general role in regulation of recombination in repetitive DNA regions and whether mitotic recombination between mating-type genes in $S$. cerevisiae is also suppressed by sister chromatid cohesion.

\section{References}

Allshire, R.C., Nimmo, E.R., Ekwall, K., Javerzat, J.P., and Cranston, G. 1995. Mutations derepressing silent centromeric domains in fission yeast disrupt chromosome segregation. Genes \& Dev. 9: 218-233.

Bernard, P. and Allshire, R. 2002. Centromeres become unstuck without heterochromatin. Trends Cell. Biol. 12: 419-424.

Bernard, P., Maure, J.F., Partridge, J.F., Genier, S., Javerzat, J.P., and Allshire, R.C. 2001. Requirement of heterochromatin for cohesion at centromeres. Science 294: 2539-2542.

Bi, X. and Broach, J.R. 1997. DNA in transcriptionally silent chromatin assumes a distinct topology that is sensitive to cell cycle progression. Mol. Cell. Biol. 17: 7077-7087.

Blat, Y. and Kleckner, N. 1999. Cohesins bind to preferential sites along yeast chromosome III, with differential regulation along arms versus the centric region. Cell 98: 249-259.

Chang, C.-R., Wu, C.-S., Hom, Y., and Gartenberg, M.R. 2005. Targeting of cohesin by transcriptionally silent chromatin. Genes \& Dev. 19: 3031-3042

Cheng, T.H. and Gartenberg, M.R. 2000. Yeast heterochromatin is a dynamic structure that requires silencers continuously. Genes \& Dev. 14: 452-463.

Ekwall, K., Nimmo, E.R., Javerzat, J.P., Borgstrom, B., Egel, R., Cranston, G., and Allshire, R. 1996. Mutations in the fission yeast silencing factors clr4+ and rik1+ disrupt the localisation of the chromo domain protein Swi6p and impair centromere function. J. Cell. Sci. 109: 2637-2648.

Ekwall, K., Cranston, G., and Allshire, R.C. 1999. Fission yeast mutants that alleviate transcriptional silencing in centromeric flanking repeats and disrupt chromosome segregation. Genetics 153: 1153-1169.

Gartenberg, M.R., Neumann, F.R., Laroche, T., Blaszczyk, M., and Gasser, S.M. 2004. Sir-mediated repression can occur independently of chromosomal and subnuclear contexts. Cell 119: 955-967.

Gerton, J. 2005. Chromosome cohesion: A cycle of holding together and falling apart. PLOS Biol. 3: E94.

Glynn, E.F., Megee, P.C., Yu, H.G., Mistrot, C., Unal, E., Koshland, D.E., DeRisi, J.L., and Gerton, J.L. 2004. Genome-wide mapping of the cohesin complex in the yeast Saccharomyces cerevisiae. PLoS Biol. 2: E259.

Gruber, S., Haering, C.H., and Nasmyth, K. 2003. Chromosomal cohesin forms a ring. Cell 112: 765-777.

Haering, C.H., Lowe, J., Hochwagen, A., and Nasmyth, K. 2002. Molecular architecture of SMC proteins and the yeast cohesin complex. Mol. Cell 9: 773-788.

Hagstrom, K.A. and Meyer, B.J. 2003. Condensin and cohesin: More than chromosome compactor and glue. Nat. Rev. Genet. 4: 520-534.

Henikoff, S. and Dalal, Y. 2005. Centromeric chromatin: What makes it unique? Curr. Opin. Genet. Dev. 15: 177-184.

Holmes, S.G. and Broach, J.R. 1996. Silencers are required for inheritance of the repressed state in yeast. Genes \& Dev. 10: 1021-1032.

Huang, C.E., Milutinovich, M., and Koshland, D. 2005. Rings, bracelet or snaps: Fashionable alternatives for Smc complexes. Philos. Trans. R Soc. Lond. B Biol. Sci. 360: 537-542.

Ivanov, D. and Nasmyth, K. 2005. A topological interaction between cohesin rings and a circular minichromosome. Cell 122: 849-860.

Karpen, G.H. and Allshire, R.C. 1997. The case for epigenetic effects on centromere identity and function. Trends Genet. 13: 489-496.

Kellum, R. and Alberts, B.M. 1995. Heterochromatin protein 1 is required for correct chromosome segregation in Drosophila embryos. J. Cell. Sci. 108: 1419-1431.

Kirchmaier, A.L. and Rine, J. 2001. DNA replication-independent silencing in S. cerevisiae. Science 291: 646-650.

Kitajima, T.S., Yokobayashi, S., Yamamoto, M., and Watanabe, Y. 2003. Distinct cohesin complexes organize meiotic chromosome domains. Science 300: 1152-1155.

Laloraya, S., Guacci, V., and Koshland, D. 2000. Chromosomal addresses of the cohesin component Mcd1p. J. Cell. Biol. 151: 1047-1056.

Lengronne, A., Katou, Y., Mori, S., Yokobayashi, S., Kelly, G.P., Itoh, T., Watanabe, Y., Shirahige, K., and Uhlmann, F. 2004. Cohesin relocation from sites of chromosomal loading to places of convergent transcription. Nature 430: 573-578.

Li, Y.C., Cheng, T.H., and Gartenberg, M.R. 2001. Establishment of transcriptional silencing in the absence of DNA replication. Science 291: 650-653.

Losada, A. and Hirano, T. 2005. Dynamic molecular linkers of the genome: The first decade of SMC proteins. Genes \& Dev. 19: 1269-1287.

Megee, P.C., Mistrot, C., Guacci, V., and Koshland, D. 1999. The centromeric sister chromatid cohesion site directs Mcdlp binding to adjacent sequences. Mol. Cell 4: 445-450.

Michaelis, C., Ciosk, R., and Nasmyth, K. 1997. Cohesins: Chromosomal proteins that prevent premature separation of sister chromatids. Cell 91: 35-45.

Milutinovich, M. and Koshland, D.E. 2003. Molecular biology. SMC complexes-Wrapped up in controversy. Science 300: 1101-1102.

Moazed, D., Rudner, A.D., Huang, J., Hoppe, G.J., and Tanny, J.C. 2004. A model for step-wise assembly of heterochromatin in yeast. Novartis Found. Symp. 259: 48-56; discussion $56-62,163-169$.

Nasmyth, K. 2005. How might cohesin hold sister chromatids together? Philos. Trans. R Soc. Lond. B Biol. Sci. 360: 483496.

Nasmyth, K. and Haering, C.H. 2005. The structure and function of SMC and kleisin complexes. Annu. Rev. Biochem. 74: 595-648.

Nonaka, N., Kitajima, T., Yokobayashi, S., Xiao, G., Yamamoto, M., Grewal, S.I., and Watanabe, Y. 2002. Recruitment of cohesin to heterochromatic regions by Swi6/HP1 in fission yeast. Nat. Cell. Biol 4: 89-93.

Peters, A.H., O'Carroll, D., Scherthan, H., Mechtler, K., Sauer, 
S., Schofer, C., Weipoltshammer, K., Pagani, M., Lachner, M., Kohlmaier, A., et al. 2001. Loss of the Suv39h histone methyltransferases impairs mammalian heterochromatin and genome stability. Cell 107: 323-337.

Rusche, L.N., Kirchmaier, A.L., and Rine, J. 2003. The establishment, inheritance, and function of silenced chromatin in Saccharomyces cerevisiae. Annu. Rev. Biochem. 72: 481516.

Sharp, J.A., Krawitz, D.C., Gardner, K.A., Fox, C.A., and Kaufman, P.D. 2003. The budding yeast silencing protein Sirl is a functional component of centromeric chromatin. Genes \& Dev. 17: 2356-2361.

Straight, A.F., Belmont, A.S., Robinett, C.C., and Murray, A.W. 1996. GFP tagging of budding yeast chromosomes reveals that protein-protein interactions can mediate sister chromatid cohesion. Curr. Biol. 6: 1599-1608.

Tanaka, T., Cosma, M.P., Wirth, K., and Nasmyth, K. 1999. Identification of cohesin association sites at centromeres and along chromosome arms. Cell 98: 847-858.

Weber, S.A., Gerton, J.L., Polancic, J.E., DeRisi, J.L., Koshland, D., and Megee, P.C. 2004. The kinetochore is an enhancer of pericentric cohesin binding. PLOS Biol. 2: E260. 


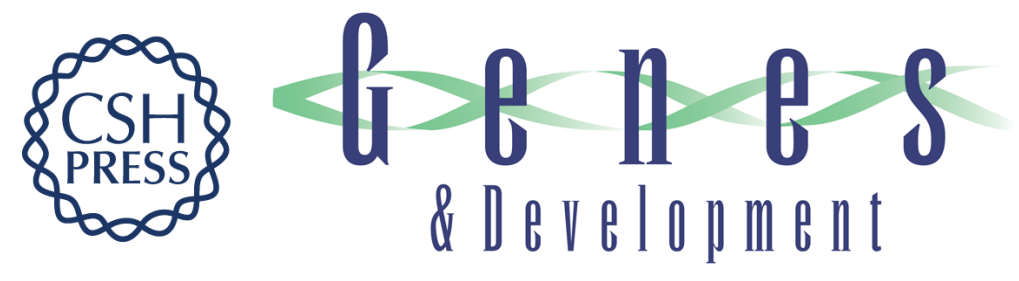

\section{Sister chromatid cohesion in silent chromatin: each sister to her own ring}

Julie Huang and Danesh Moazed

Genes Dev. 2006, 20:

Access the most recent version at doi:10.1101/gad.1398106
Related Content Targeting of cohesin by transcriptionally silent chromatin Chuang-Rung Chang, Ching-Shyi Wu, Yolanda Hom, et al. Genes Dev. December , 2005 19: 3031-3042
References This article cites 40 articles, 16 of which can be accessed free at: http://genesdev.cshlp.org/content/20/2/132.full.html\#ref-list-1
Articles cited in:
http://genesdev.cshlp.org/content/20/2/132.full.html\#related-urls
License Email Alerting
Service $\begin{aligned} & \text { Receive free email alerts when new articles cite this article - sign up in the box at the top } \\ & \text { right corner of the article or click here. }\end{aligned}$

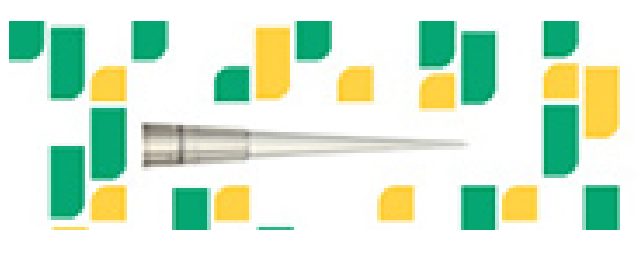

Focused on your science. 